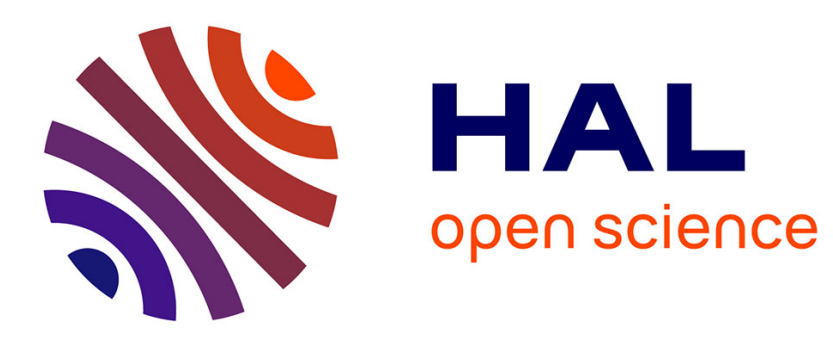

\title{
Fault diagnosis in railway track circuits using Dempster-Shafer classifier fusion
}

Latifa Oukhellou, Alexandra Debiolles, Thierry Denoeux, Patrice Aknin

\section{To cite this version:}

Latifa Oukhellou, Alexandra Debiolles, Thierry Denoeux, Patrice Aknin. Fault diagnosis in railway track circuits using Dempster-Shafer classifier fusion. Engineering Applications of Artificial Intelligence, 2010, 23 (1), pp.117-128. 10.1016/j.engappai.2009.06.005 . hal-00467941

\section{HAL Id: hal-00467941 \\ https://hal.science/hal-00467941}

Submitted on 29 Mar 2010

HAL is a multi-disciplinary open access archive for the deposit and dissemination of scientific research documents, whether they are published or not. The documents may come from teaching and research institutions in France or abroad, or from public or private research centers.
L'archive ouverte pluridisciplinaire HAL, est destinée au dépôt et à la diffusion de documents scientifiques de niveau recherche, publiés ou non, émanant des établissements d'enseignement et de recherche français ou étrangers, des laboratoires publics ou privés. 


\title{
Fault diagnosis in railway track circuits using Dempster-Shafer classifier fusion
}

\author{
Latifa Oukhellou $^{\mathrm{a}, \mathrm{b}^{*}}$, Alexandra Debiolles ${ }^{\mathrm{c}}$, Thierry Denœux ${ }^{\mathrm{d}}$, Patrice Aknin ${ }^{\mathrm{b}}$ \\ ${ }^{a}$ Certes, Université Paris XII, 61 Av du Gal de Gaulle 94110 Créteil France \\ ${ }^{\mathrm{b}}$ LTN, Institut National de recherche sur les Transports et leur Sécurité, 2 Av Malleret Joinville 94114 Arcueil France \\ ${ }^{\mathrm{c}} \mathrm{SNCF}$, ingénierie IG SF 43, 4 et 6 Av François Mitterrand, 93574 La plaine St Denis France \\ ${ }^{\mathrm{d}}$ Heudiasyc, Université de Technologie de Compiègne, UMR CNRS 6599, BP 20529, 60205 Compiègne France
}

$\operatorname{xxxXXXXXXXXXXXX}$

\begin{abstract}
This paper addresses the problem of fault detection and isolation in railway track circuits. A track circuit can be considered as a large-scale system composed of a series of trimming capacitors located between a transmitter and a receiver. A defective capacitor affects not only its own inspection data (short circuit current) but also the measurements related to all capacitors located downstream (between the defective capacitor and the receiver). Here, the global fault detection and isolation problem is broken down into several local pattern recognition problems, each dedicated to one capacitor. The outputs from local neural network or decision tree classifiers are expressed using Dempster-Shafer theory and combined to make a final decision on the detection and localization of a fault in the system. Experiments with simulated data show that correct detection rates over $99 \%$ and correct localization rates over $92 \%$ can be achieved using this approach, which represents a major improvement over the state of the art reference method.
\end{abstract}

Keywords: Classifier Fusion, Belief Functions, Neural Networks, Evidence Theory, Transferable Belief Model, Fault Detection and Isolation, Pattern Recognition.

*Corresponding author

E-mail adress : oukhellou@,univ-paris12.fr, fax : +33 (0)1 45925501

LTN, Institut National de recherche sur les Transports et leur Sécurité, Le Descartes 2 - 2 rue de la Butte Verte. 93166

Noisy-le-Grand Cedex 


\section{Introduction}

Complex industrial systems need to be monitored continuously to detect dysfunctions and maintain a good quality of service. In the railway domain, the infrastructure is often inspected by instrumented vehicles in order to ensure a high level of safety and availability within a predictive maintenance framework. For some applications, signals recorded during inspection runs are manually analyzed by maintenance experts and technicians in order to identify anomalies. In this case, there is a need for automatic fault detection and isolation (FDI) systems, which can reduce the time consumed by the analysis phase and improve the diagnosis performances. The aim of such systems is to detect the occurrence of a fault based on recorded measurements and then determine the exact nature and location of the fault. Maintainers are thus provided with an accurate and systematic analysis of recordings that allows them to schedule preventive maintenance appropriately.

Various approaches can be adopted in order to build an automatic FDI system, depending on the available knowledge of the system under study. In the so-called model-based approach, an accurate model of the system is typically assumed to be available. Several approaches have been proposed to exploit analytical redundancy, including parity space equations, state observers and process identification [17] [21] [24] [30] [33]. In contrast, the pattern recognition approach to fault diagnosis uses only a set of historical data containing representative measurements acquired under various normal and abnormal conditions [33] [11]. In most cases, an initial pre-processing stage, referred to as feature extraction, maps data from the measurement space to a reduced feature space relevant to the FDI task. Machine learning techniques (such as decision trees, neural networks or support vector machines [14][4][23]) then capture the relationships between feature values and system states, based on learning data. To ensure good generalization, this approach requires some knowledge of the system to extract relevant features, as well as a large and exhaustive labelled database covering most of the situations that can be encountered in practice. A comparison between the model-based and pattern recognition approaches is given in [33], and an approach to fault diagnosis based on trend analysis is presented in [28].

This paper presents a method for FDI in railway track circuits based on a statistical pattern recognition approach. Track circuits generally use a scheduled maintenance regime: inspection is carried out on every track circuit periodically (typically, every 15 days) and inspection recordings are analyzed visually in order to detect major defects. Recent work on fault detection and diagnosis for railway track circuits has been published in [3]. The authors proposed a neuro-fuzzy system that makes it possible to detect and diagnose the most common track circuit failures in a laboratory test rig. The diagnosis system proposed here differs since it uses a train-based approach and it is dedicated to detect different kind of defects.

A track circuit is composed of a series of trimming capacitors connected between the two rails [1] [18]. Part of the difficulty of FDI task for this system arises from the fact that the presence of a fault in one subsystem (trimming capacitor) influences the inspection signal of other subsystems located downstream 
(between the defective capacitor and the receiver). This is depicted schematically in Figure 1, where $S_{1}, \ldots, S_{N}$ represent the $N$ trimming capacitors, and $I_{i}$ denotes the inspection signal (measurement) for capacitor $S_{i}$. A fault in $S_{i}$ influences the inspection signals corresponding to all capacitors between $S_{i}$ and $S_{N}$. Consequently, the fault isolation task must take into account the spatial relationship between subsystems: an abnormal measurement from one subsystem may indicate a fault in that subsystem or in any of the subsystems located upstream of it.

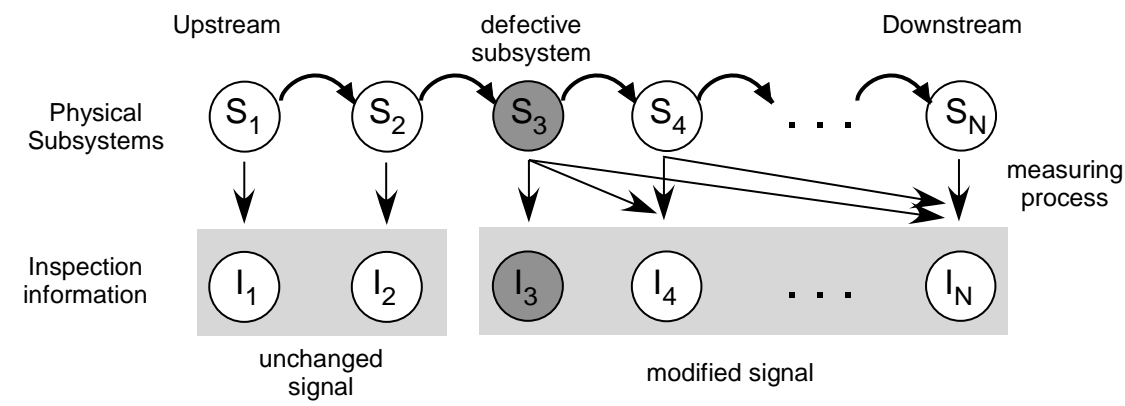

Fig.1. Spatial relationship between subsystems: a defective subsystem $\left(\mathrm{S}_{3}\right)$ influences the inspection signals $I_{3}, I_{4}, \ldots, I_{N}$ related to downstream subsystems.

The approach presented here uses machine learning (neural networks, decision trees) and information fusion techniques to perform fault detection and isolation. Each capacitor is classified as fault-free or defective using a neural network or decision tree classifier and the local decisions are combined to make a final decision regarding the presence and location of a fault.

Training a neural network classifier involves minimizing the discrepancy between the neural network outputs and target outputs encoding class membership of training patterns. Consequently, an output coding scheme has to be chosen. Two such schemes will be tested and evaluated. In the first one, only the target output related to the defective subsystem is set to 1 (the others being set to 0 ). This scheme may be referred to as local coding. Alternatively, the target outputs of both the defective subsystem and the subsystems located downstream of it may be set to 1; in this case, the coding is said to be distributed. Each classifier thus provides information on the presence of a fault either in a specific subsystem (local coding) or in any of the subsystems located upstream (distributed coding).

A large number of methods for classifier fusion have been proposed. For many applications, it has been shown that combining classifiers can yield performance improvements [2] [41] [16] [27]. Depending on the information provided by the individual classifiers, three categories of fusion methods can be distinguished [25] [42]:

- When each classifier produces a single class label output (crisp label), the two most representative approaches are majority voting [26], and the behaviour-knowledge space (BKS) method [20]. 
- The second group of fusion methods is of the rank level decision type, i.e., the output of each classifier is a subset of possible labels ranked by plausibility. The fusion strategies applied on outputs of this type are based on class set reduction, class set reordering or a combination of both [19]. The first approach aims at reducing the set of considered classes while the objective of the second one is to place the true class as close as possible to the top.

- The third and largest group of fusion methods operates on classifiers that produce so-called soft labels in the range [0,1]. These methods can be based on Bayesian Probability theory [27] [34], Possibility Theory [31] [13] [44] or the theory of belief functions, also referred to as DempsterShafer or evidence theory [11] [12] [5] [22] [43]. The method investigated here is based on the latter approach, which provides a flexible framework for handling uncertainty and managing the conflict between several classifiers.

The rest of the paper is organized as follows. Section 2 describes the application under study and introduces the diagnosis problem in greater detail. The necessary notions of Dempster-Shafer theory are then recalled in Section 3. A system for fault detection and isolation in track circuits based on classifier fusion in the Dempster-Shafer framework is then presented in Section 4. Experimental results are finally reported in Section 5, and Section 6 concludes the paper.

\section{Case study}

The application considered in this paper concerns FDI in railway track circuits. This device will first be described in Section 2.1, and the problem addressed will be exposed in Section 2.2. An overview of the proposed FDI method will then be presented in Section 2.3.

\subsection{Track circuit principle}

The track circuit is an essential component of the automatic train control system. Its main function is to detect the presence or absence of vehicle traffic within a specific section of railway track. The signalling system uses the occupation of track section to protect trains from coming into conflict. On French high speed lines, the track circuit is also a fundamental component of the track/vehicle transmission system. It uses a specific carrier frequency to transmit coded data to the train, for example the maximum authorized speed on a given section on the basis of safety constraints.

The railway track is divided into different sections. Each one of them has a specific track circuit (Figure 2) consisting of the following components:

- A transmitter connected to one of the two section ends, which delivers a frequency modulated alternating current;

- The two rails that can be considered as a transmission line; 
- At the other end of the track section, a receiver that essentially consists of a trap circuit used to avoid the transmission of information to the neighbouring section;

- Trimming capacitors connected between the two rails at constant spacing to compensate for the inductive behaviour of the track. Electrical tuning is then performed to limit the attenuation of the transmitted current and improve the transmission level. The number of compensation points depends on the carrier frequency and the length of the track section.

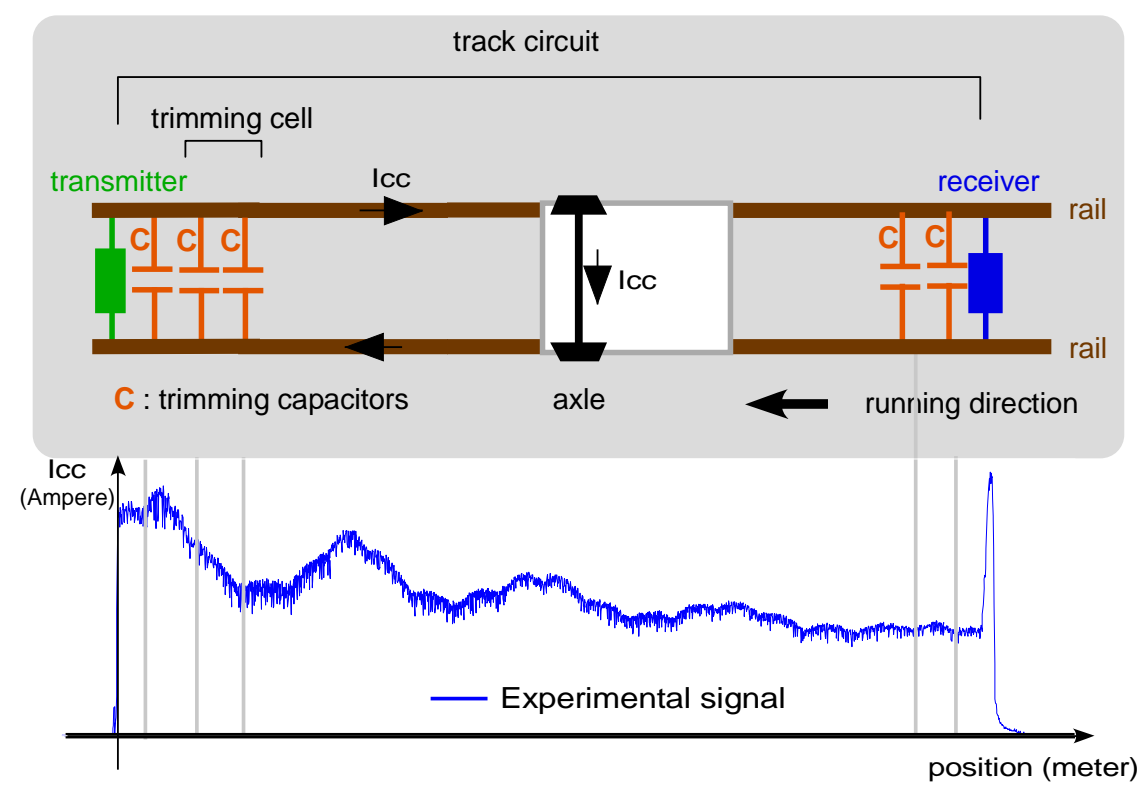

Fig. 2. Diagram of a track circuit and example of an inspection signal.

The rails themselves are part of the track circuit, and a train is detected when its wheels and axles shortcircuit the track. The presence of a train in a given section induces the loss of track circuit signal due to shorting by train wheels. The drop of the received signal below a preset threshold indicates that the section is occupied. In order to make the transmitted information specific to each track section and to minimize the influence of both longitudinal interference and transverse crosstalk, four frequencies are used for adjacent and parallel track circuits. Neighbouring track circuits are also isolated electrically by using tuned circuits (capacitor and inductance) on both the transmitter and the receiver.

\subsection{Problem description}

The different parts of the track circuit are subject to malfunctions (due to aging, atmospheric conditions or track maintenance operations) that must be detected as soon as possible in order to maintain the system at the required safety and availability levels. In the most extreme cases, such malfunctions can cause a significant attenuation of the transmitted signal which may induce signalling problems. The purpose of 
system diagnosis is to inform maintainers about track circuit failures, thus ensuring that the quality of transmitted information remains high.

A vehicle-based fault diagnosis system can be developed by equipping an inspection vehicle with a sensor in front of the first axle. The carrier current level of the short-circuit current (Icc) is picked up by the sensor coils and recorded at each position of the train, while the track circuit is shunted by the inspection train itself (Figure 2).

This paper will focus on trimming capacitor faults that affect capacitor internal resistance. The deviation from the ideal behavior of the capacitor, mainly due to dielectric aging, is taken into account by introducing a serial resistance $r$ that is null (or weak) when the capacitor is healthy and increases when it is defective. An electrical model has been developed that is able to perform realistic simulations of the system including a large variety of dysfunctions [1] [18] [9]. Figure 3 shows examples of Icc signals simulated along a $1500 \mathrm{~m}$ track circuit: one of them corresponds to an absence of fault, while the others correspond to a defective $9^{\text {th }}$ capacitor. The aim of the fault detection and isolation system is to detect the operating mode of the track circuit by analyzing the measurement signal.

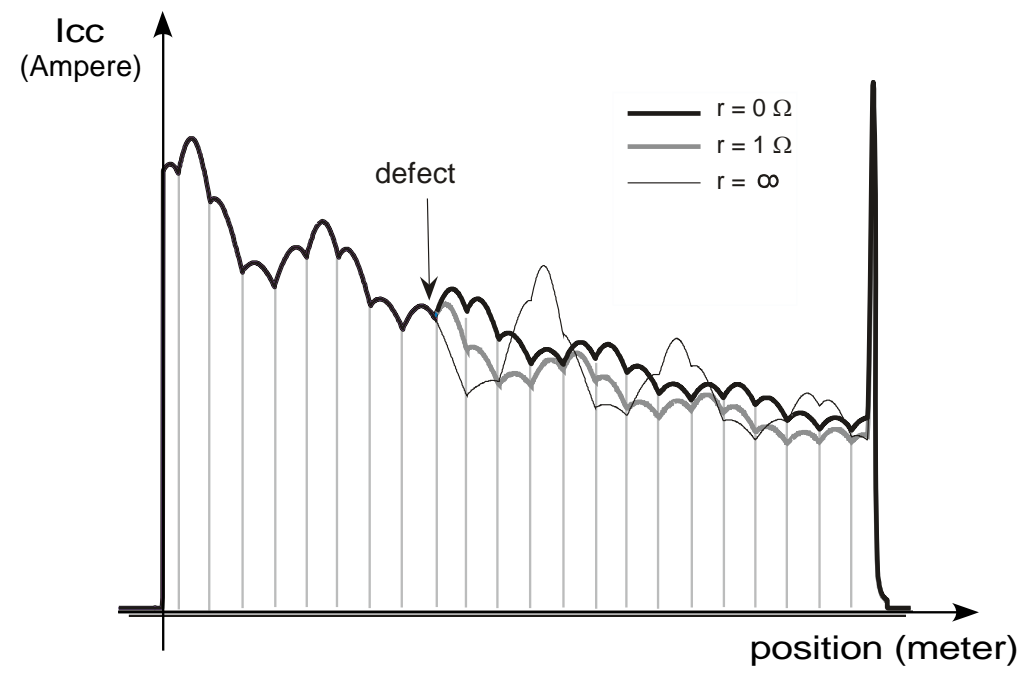

Fig. 3. Example of simulated Iccsignal without fault $(\mathrm{r}=0 \Omega)$ and with a defective $9^{\text {th }}$ capacitor $(\mathrm{r}=1 \Omega$ and $\mathrm{r}=\infty)$.

\subsection{Overview of the diagnosis method}

The proposed method is based on the following two observations (see Figure 3):

- The inspection Icc signal has a specific pattern, which is a succession of arches also called catenary curves, each one corresponding to a trimming capacitor; an arch can be approximated by a quadratic polynomial. 
- The presence of a fault in the system only affects the shape of the arches between the fault and the receiver leaving the signal upstream (between the transmitter and the fault) unchanged.

The proposed method consists in extracting features from the Icc signal, and training one classifier for each trimming capacitor. Elementary classifier outputs are then represented using the formalism of belief functions and combined. Finally, a decision regarding the presence and location of a fault is made.

\section{Dempster-Shafer theory}

This section will provide a brief account of the fundamental notions of the Dempster-Shafer theory of belief functions, also referred to as Evidence Theory. This uncertain reasoning framework was initiated by Dempster [10] and developed by Shafer [35]. It can be seen as an extension of Bayesian Probability Theory. A particular interpretation of Dempster-Shafer theory has been proposed by Smets [37], under the name of the Transferable Belief Model (TBM). The TBM has a two-level structure composed of

- a credal level where beliefs are entertained, and

- a pignistic level where decisions are made.

In this section, we shall define only the concepts used in the diagnosis method introduced below. Further details can be found in [36] [37] [38] [39][32].

\subsection{Representation of beliefs: the credal level}

Let $\Theta$ denote the set of possible answers to a given problem, called the frame of discernment. Here, $\Theta$ will be assumed to be finite:

$$
\Theta=\left\{\theta_{1}, \theta_{2}, \ldots, \theta_{n}\right\} .
$$

A basic belief assignment (BBA) is a function $m$ from $2^{\Theta}$ to $[0,1]$ that assigns a "mass of belief" to each subset $A$ of the frame of discernment $\Theta$, such that:

$$
\sum_{A \subseteq \Theta} m(A)=1 .
$$

The basic belief mass $m(A)$ represents a measure of the belief that is assigned to subset $A \subseteq \Theta$, given the available evidence, and that cannot be committed to any strict subset of $A$. Every $A \subseteq \Theta$ such that $m(A)>0$ is called a focal set. A $B B A$ such that $m(\varnothing)=0$, where $\varnothing$ denotes the empty set, is said to be normal. The quantity $m(\varnothing)$ can be interpreted as that part of belief that is committed to the assumption that none of the hypotheses in $\Theta$ might be true (open-world assumption). When $m(\Theta)=1, m$ is called a vacuous BBA: it represents total ignorance regarding the question under consideration. 
Example 1: Let $\Theta=\left\{\theta_{1}, \theta_{2}, \theta_{3}\right\}$ denote the possible states of a system under study, where $\theta_{1}$ is the normal state, and the other two states are faulty. Assume that we have some evidence that the system is in a faulty state (but we do not know which one), and we have a degree of confidence of 0.8 in this piece of evidence. This information can be represented as the following BBA:

$$
\begin{aligned}
m_{1}\left(\left\{\theta_{2}, \theta_{3}\right\}\right) & =0.8 \\
m_{1}(\Theta) & =0.2 .
\end{aligned}
$$

We note that the mass 0.8 is attached to $\left\{\theta_{2}, \theta_{3}\right\}$ because the piece of evidence only tells us that the system is in a faulty state, without pointing specifically to $\theta_{2}$ or $\theta_{3}$. The remaining mass of 0.2 is not assigned to $\theta_{1}$ as no evidence points to that particular hypothesis. It remains uncommitted and attached to the whole frame of discernment.

\subsection{Combination of several BBAs}

To combine several BBAs defined on the same frame of discernment, Smets introduced the conjunctive rule of combination, also referred to as the unnormalized Dempster's rule [39]. For this rule to be used, the different BBAs must be based upon independent pieces of evidence. Let $m_{1}$ and $m_{2}$ be two BBAs. The BBA that results from their conjunctive combination, denoted by $m_{1} \cap m_{2}$, is defined for all $A \subseteq \Theta$ as:

$$
m_{1} \cap m_{2}(A)=\sum_{B, C \subseteq \Theta: B \cap C=A} m_{1}(B) m_{2}(C) .
$$

This rule is commutative and associative. When combining $n$ BBAs $m_{1}, \ldots, m_{n}$, we may thus either use (3) iteratively, or use the following direct formula:

$$
m_{1} \cap \ldots \cap m_{n}(A)=\sum_{B_{1} \cap \ldots \cap B_{n}=A} m_{1}\left(B_{1}\right) \ldots m_{n}\left(B_{n}\right) .
$$

The mass $m(\varnothing)$ assigned to the empty set may be interpreted as a measure of conflict between the two sources.

Example 2: Continuing Example 1, assume that we receive another independent piece of evidence telling us that the system is not in state $\theta_{2}$, with confidence 0.6. This evidence may be represented by the following BBA:

$$
\begin{aligned}
m_{2}\left(\left\{\theta_{1}, \theta_{3}\right\}\right) & =0.6 \\
m_{2}(\Theta) & =0.4 .
\end{aligned}
$$

Combining $m_{1}$ and $m_{2}$ using (3) yields the BBA $m=m_{1} \cap m_{2}$ defined as follows: 


$$
\begin{aligned}
m\left(\left\{\theta_{3}\right\}\right) & =0.8 \times 0.6=0.48 \\
m\left(\left\{\theta_{2}, \theta_{3}\right\}\right) & =0.8 \times 0.4=0.32 \\
m\left(\left\{\theta_{1}, \theta_{3}\right\}\right) & =0.2 \times 0.6=0.12 \\
m(\Theta) & =0.2 \times 0.4=0.08 .
\end{aligned}
$$

We observe that the masses still sum to one.

\subsection{Discounting}

The reliability of a source of information can be taken into account by discounting the original BBA $m$ by a discount rate $1-\alpha$, where $\alpha$ is a coefficient between 0 and 1 . The smaller the reliability, the larger is the discount rate. The resulting $\mathrm{BBA}^{\alpha} m$ is defined by [35] [40]:

$$
\begin{aligned}
& { }^{\alpha} m(A)=\alpha m(A), \quad \forall A \subset \Theta, \\
& { }^{\alpha} m(\Theta)=1-\alpha[1-m(\Theta)] .
\end{aligned}
$$

In this way, part of the total mass of belief is transferred to $\Theta$, resulting in a less informative BBA. The coefficient $\alpha$ represents the degree of belief that the source is reliable [40] [29]. If $\alpha=1$, we have full confidence in the source and the belief function is unchanged. The closer $\alpha$ is to zero, the closer the resulting BBA is to the vacuous belief function $(m(\Theta)=1)$, meaning that the information provided by the source is discarded. Several methods have been proposed to learn the optimal value of $\alpha$ from data [15] [29].

Example 3: Assume that we are about to toss a coin, and we represent our belief regarding the outcome on the frame $\Theta=\{$ Head,Tails $\}$. If the coin is fair, then our belief on $\Theta$ can be represented by the following BBA:

$$
\begin{aligned}
& m(\{\text { Head }\})=0.5 \\
& m(\{\text { Tails }\})=0.5 .
\end{aligned}
$$

Such a BBA, whose focal sets are singletons, is equivalent to a probability distribution and is called a Bayesian BBA. Assume now that we are not sure that the coin is fair, and we only have a degree of belief equal to 0.7 in this hypothesis. Then, the above BBA should be discounted by a rate $1-\alpha=1-0.7=0.3$. The discounted BBA is

$$
\begin{aligned}
{ }^{\alpha} m(\{\text { Head }\}) & =0.7 \times 0.5=0.35 \\
{ }^{\alpha} m(\{\text { Tails }\}) & =0.7 \times 0.5=0.35 \\
{ }^{\alpha} m(\Theta) & =0.3 .
\end{aligned}
$$


We observe that part of the mass has been transferred to the frame of discernment, which reflects partial ignorance of the probabilities.

\subsection{Decision-making: the pignistic level}

The TBM is based on a two-level structure: the credal level where beliefs are entertained and the pignistic $^{l}$ level where BBAs ar converted into probability distributions are used to make decisions according to the principle of maximal expected utility [36] [37].

When a decision has to be made, the BBAs are transformed into probabilities. For this purpose, we build a pignistic probability function BetP from the BBA $m$, using the pignistic transformation defined as [36]:

$$
\operatorname{Bet} P(\theta)=\sum_{\{B \subseteq \Theta, \theta \in B\}} \frac{1}{|B|} \frac{m(B)}{1-m(\varnothing)}, \forall \theta \in \Theta
$$

where $|B|$ denotes the cardinality of $B$ and it is assumed that $m(\varnothing)<1$.

This definition is based on the idea that, in the absence of additional information, $m(B)$ should be equally distributed between the components of $B$, for all $B \subseteq \Theta$. The pignistic probability is a classical probability measure that can be used for decision making using standard Bayesian decision theory. A detailed discussion on this concept can be found in [36].

Example 4: Let us come back to the BBA $m$ of Example 2. Its pignistic probability function is:

$$
\begin{aligned}
& \operatorname{BetP}\left(\left\{\theta_{1}\right\}\right)=\frac{0.12}{2}+\frac{0.08}{3} \simeq 0.09 \\
& \operatorname{Bet} P\left(\left\{\theta_{2}\right\}\right)=\frac{0.32}{2}+\frac{0.08}{3} \simeq 0.19 \\
& \operatorname{BetP}\left(\left\{\theta_{3}\right\}\right)=0.48+\frac{0.32}{2}+\frac{0.12}{2}+\frac{0.08}{3} \simeq 0.73
\end{aligned}
$$

\section{The diagnostic system}

The whole architecture of the proposed diagnosis system is sketched in Figure 4. In this figure, the system $\Sigma$ represents a track circuit, and the $N$ subsystems $S_{1}, \ldots, S_{N}$ are the trimming capacitors. As explained in Section 2, the objective of the application is to detect a fault in any of these capacitors, and to determine the position of the defective capacitor if a fault has been detected. It is assumed that there is at most one defective capacitor. In Figure $4, I_{i}$ represents the measurement data corresponding to capacitor $S_{i}$. The vector of measurement data $I_{1}, \ldots, I_{N}$ consists in the features extracted from the Icc curve as displayed

\footnotetext{
${ }^{1}$ From the Latin word "pignus" meaning "a bet".
} 
in Figure 2. The arrows between $S_{3}$ and $I_{3}, \ldots, I_{N}$ represent the fact that a fault in capacitor $S_{3}$ (for instance) influences the measurement data (i.e., the shape if catenary curves) related to all capacitors downstream.

As shown in Figure 4, the proposed system is composed of $N$ local classifiers $D_{l}, \ldots, D_{N}$. Two distinct learning tasks can be assigned a priori to each local classifier $D_{i}$ : it can be trained to predict either if capacitor $S_{i}$ is faulty, or if any of the upstream capacitors is faulty. These two approaches will be hereafter referred to as local and distributed coding, respectively. As they both seem reasonable, these two approaches will be investigated below.

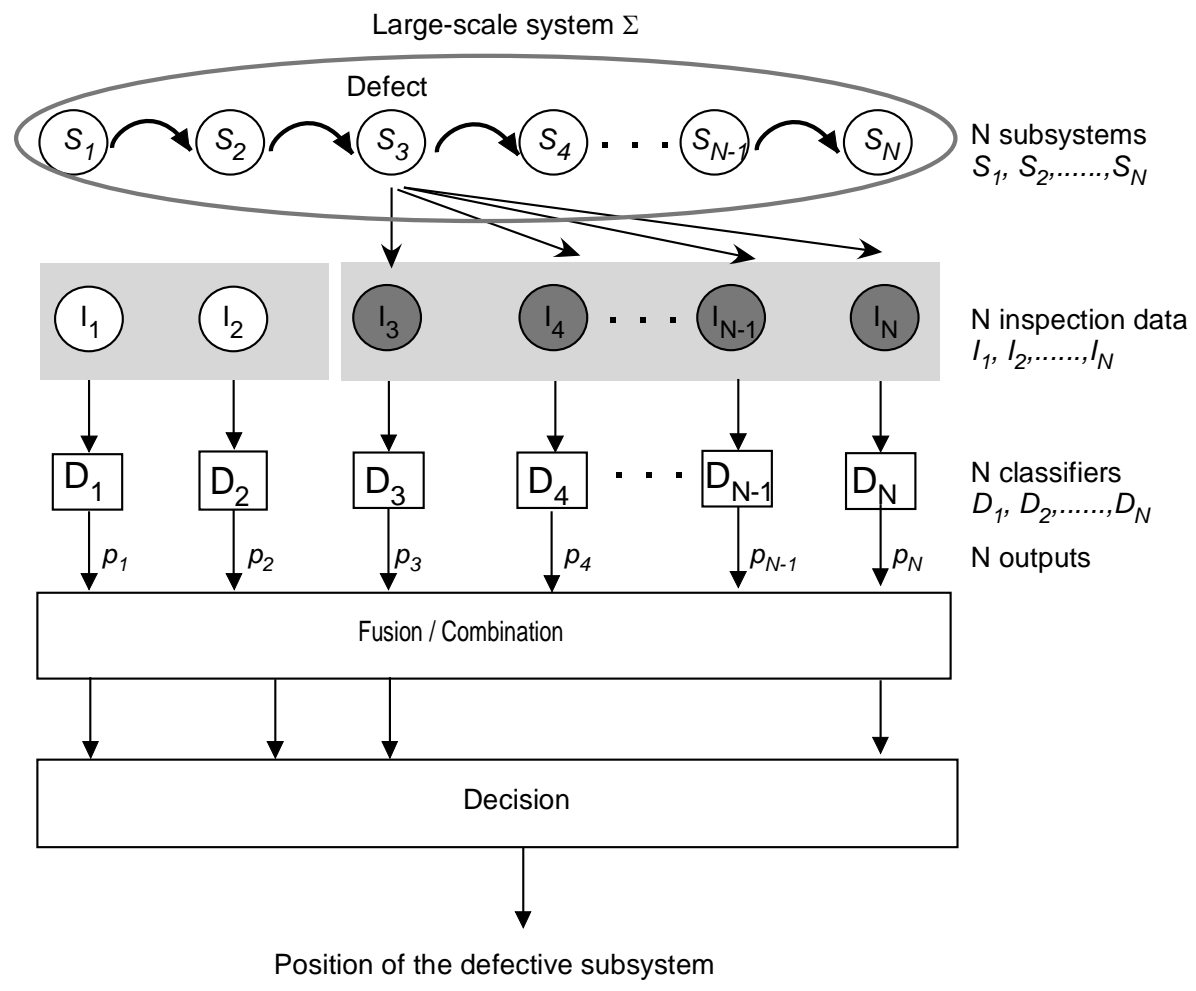

Fig. 4. Principle of the fault detection and isolation method.

Each classifier $D_{i}$ receives feature values extracted from raw inspection data, and computes a probability $p_{i}$. Depending on the coding chosen, this output is converted into a Dempster-Shafer mass function $m_{i}$. The $N$ mass functions are then pooled into a combined mass function m using (3)-(4), and a decision regarding the presence and location of the fault is made based on pignistic probabilities (6).

In the rest of this section, we will first describe the feature space and the decision space of each local classifier in Subsections 4.1, and 4.2, respectively. The fusion process will then be described in Subsections 4.3, and the final decision procedure will be presented in Subsection 4.4. Details regarding the training of classifiers will be presented with experimental results in Section 5.

\subsection{Feature extraction}

As explained in Section 2.2, the inspection data take the form of a Icc curve as shown in Figure 3. This constitutes the measurement space. The Icc curve is composed of arched curve segments. Each of these 
arches corresponds to a trimming capacitor. To obtain a compact representation of the data, each arch was approximated by a quadratic polynomial and the three coefficients $\left(a_{i}, b_{i}, c_{i}\right)$ of each polynomial were used as features (see Figure 5). The whole Icc curve was thus described by a total of $3 \mathrm{~N}$ features.

As a fault in capacitor $S_{i}$ affects the inspection data downstream, each classifier $D_{i}$ has to be provided with upstream information. As shown in Figure 5, the inputs of classifier $D_{i}$ are the $3 i$ parameters corresponding to the $i$ arches between the transmitter and the $i^{\text {th }}$ trimming capacitor. The features spaces of the classifiers are thus nested.

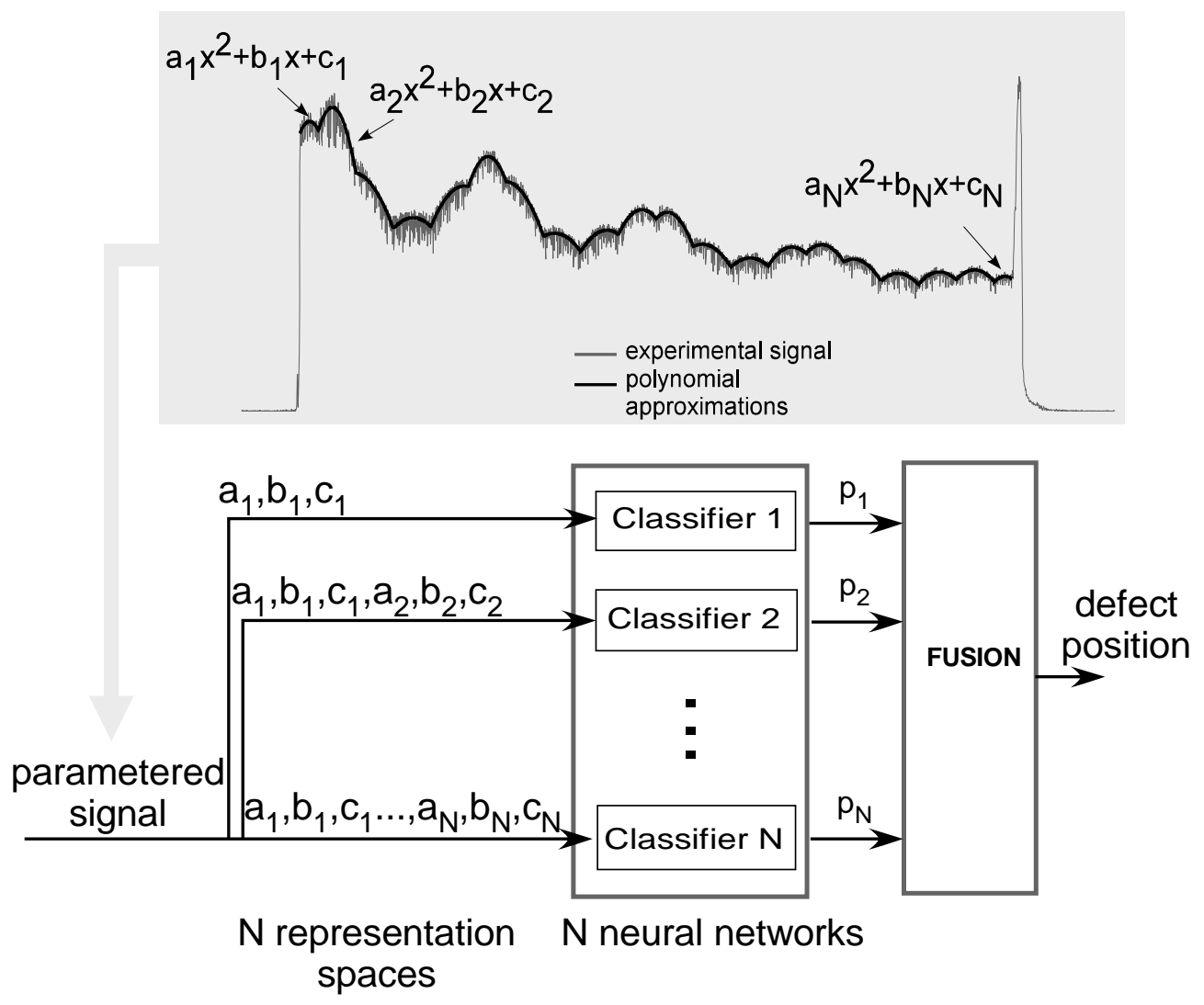

Fig. 5. Feature extraction.

\subsection{Decision Spaces of Local Classifiers}

In this paper, we consider neural network or decision tree classifiers, whose outputs are interpreted as probabilities. As explained above, each local classifier $D_{i}$ can be trained either to detect a fault in capacitor $S_{i}$, or to detect a fault in any capacitor between $S_{l}$ and $S_{i}$. When neural networks are used as classifiers, the nature of the learning task will affect the coding of neural network outputs, as shown in Figure 6. Assume that a fault has occurred in capacitor $S_{i}$ (with $i=3$ in Figure 6). Two cases will be considered:

- If local coding is chosen (coding 1 in Figure 6), the desired output for classifier $D_{i}$ is set to 1 and the desired outputs of all other classifiers are set to 0 . 
- In the case of distributed coding (coding 2 in Figure 6), the desired outputs of classifiers $D_{j}$ with $j \geq i$ are set to 1 and the desired outputs of all other classifiers are set to 0 .

Distributed coding seems a priori to be more appropriate to account for the spatial relationship between subsystems. However, both solutions will be studied and experimented in this paper.

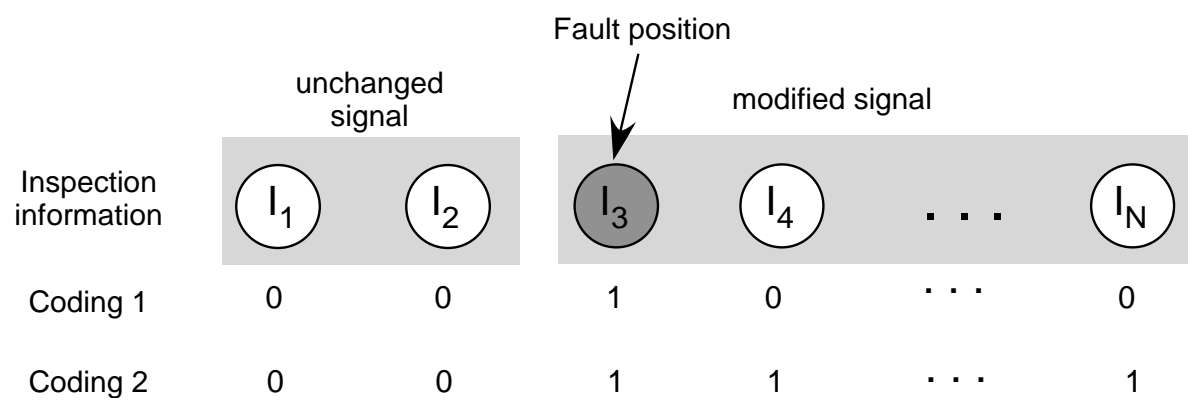

Fig. 6. The two output coding schemes used to train the $N$ classifiers when capacitor $S_{3}$ is defective. Using local coding (coding 1), only the desired output of classifier $D_{3}$ is set to 1.Using distributed coding (coding 2), the desired outputs of all classifiers $D_{j}$ with $j \geq 3$ are set to 1 .

\subsection{Classifier Fusion}

As classifiers $D_{i}(i=1, \ldots, N)$ process only local information, their outputs have to be combined to reach a final decision regarding the presence and location of a defective capacitor. Here, the classifier outputs will are expressed in the Dempster-Shafer framework and combined using Dempster's rule (3)-(4).

As indicated in Section 3, to build a Dempster-Shafer model we first need to define the frame of discernment. Here, it will be the set $\Theta=\{1, \ldots, N, N+1\}$, where $N$ is the number of capacitors in the track circuit. Each singleton $\{i\}, i=1, \ldots, N+1$ corresponds to a possible position of the fault. The virtual position $N+1$ corresponds to the absence of fault.

The conversion of classifier outputs into mass functions and their combination will be presented below, first in the case of local coding, and then in the case of distributed coding.

\subsubsection{Classifier fusion with local coding}

Using local coding, if the output of classifier $D_{i}$ is 1 , the fault is considered to be in capacitor $S_{i}$. Otherwise, there is either a fault in some capacitor $S_{j}$ with $j \neq i$ or no fault. The output from the classifier $D_{i}$ can thus be represented as BBA $m_{i}$ with two focal sets: the singleton $\{i\}$ and its complement $\Theta \backslash\{\mathrm{i}\}$ :

$$
\begin{array}{ll}
m_{i}(\{i\}) & =p_{i} \\
m_{i}(\Theta \backslash\{i\}) & =1-p_{i},
\end{array}
$$

where $p_{i} \in[0,1]$ is the output of classifier $D_{i}$.

The combination of the $N$ BBAs $m_{l}, \ldots, m_{N}$ using the unnormalized Dempster's rule (4) yields a BBA $m$ with $N+1$ focal sets: the singletons and the empty set. This BBA has the following expression: 


$$
\begin{array}{ll}
m(\{i\}) & =p_{i} \prod_{j \neq i}\left(1-p_{j}\right) \quad \forall i=1, \cdots, N \\
m(\{N+1\}) & =\prod_{i=1}^{N}\left(1-p_{i}\right) \\
m(\varnothing) & =1-\sum_{i=1}^{N+1} m(\{i\}) .
\end{array}
$$

Let us now assume that we discount each classifier output by a rate $1-\alpha_{i}$ with $0 \leq \alpha_{i} \leq 1$ (see Section 3.3). As will be explained in Section 5, the discount rate can be determined as function of the classifier error. The BBA $m_{i}$ representing the output of classifier $D_{i}$ then becomes:

$$
\begin{array}{ll}
m_{i}(\{i\}) & =\alpha_{i} p_{i} \\
m_{i}(\Theta \backslash\{i\}) & =\alpha_{i}\left(1-p_{i}\right) \\
m_{i}(\Theta) & =1-\alpha_{i} .
\end{array}
$$

Combining these $N$ BBAs using the unnormalized Dempster's rule now gives a BBA $m$ defined as follows:

$$
\begin{array}{ll}
m(\{i\}) & =\alpha_{i} p_{i} \prod_{j \neq i}\left[\alpha_{j}\left(1-p_{j}\right)+\left(1-\alpha_{j}\right)\right], \quad \forall i=1, \cdots, N \\
m(\{N+1\}) & =\prod_{i=1}^{N} \alpha_{i}\left(1-p_{i}\right) \\
m(A) & =\prod_{i \notin A} \alpha_{i}\left(1-p_{i}\right) \quad \prod_{i \in A}\left(1-\alpha_{i}\right), \quad \forall A \subset \Theta, N+1 \in A, 1<|A|<N, \\
m(\Theta) & =\prod_{i=1}^{N}\left(1-\alpha_{i}\right),
\end{array}
$$

the rest of the mass being assigned to the empty set. By convention, the products in the above expressions vanish when the indices of the coefficients are negative or 0.

\subsubsection{Classifier fusion with distributed coding}

Assume that distributed coding was used, and the output of classifier $D_{i}$ is equal to 0 . This means that no fault was detected between $S_{1}$ and $S_{i}$ : consequently, there is either a fault between $S_{i+1}$ and $S_{N}$, or no fault. On the contrary, if the output of classifier $D_{i}$ is equal to 1 , it means that a fault was detected between $S_{l}$ and $S_{i}$. The information provided by classifier $D_{i}$ can therefore be represented by the following BBA:

$$
\begin{aligned}
& m_{i}([1, i])=p_{i} \\
& m_{i}([i+1, N+1])=1-p_{i} .
\end{aligned}
$$

where, as before, $p_{i} \in[0,1]$ denotes the output of classifier $D_{i}$, and $[1, i]$ denotes the set of integers between 1 and $i$.

The combination of $m_{1}, \ldots, m_{N}$ using Dempster's rule (4) yields the following BBA: 


$$
\begin{aligned}
& m(\{i\}) \quad=\prod_{j=1}^{i-1}\left(1-p_{j}\right) \prod_{k=i}^{N} p_{k}, \quad \forall i=1, \cdots, N \\
& m(\{N+1\})=\prod_{i=1}^{N}\left(1-p_{i}\right) \\
& m(\varnothing) \quad=1-\sum_{i=1}^{N+1} m(\{i\}) .
\end{aligned}
$$

If, as before, we now discount each BBA representing the output of classifier $D_{i}$ by a rate $1-\alpha_{i}$, the BBA $m_{i}$ given by the $i^{\text {th }}$ classifier becomes:

$$
\begin{aligned}
m_{i}([1, i]) & =\alpha_{i} p_{i} \\
m_{i}([i+1, N+1]) & =\alpha_{i}\left(1-p_{i}\right) \\
m_{i}(\Theta) & =1-\alpha_{i} .
\end{aligned}
$$

Combining these BBAs using the unnormalized Dempster's rule now yields:

$$
\begin{array}{lll}
m(\{1\}) & =\alpha_{1} p_{1} \prod_{j=2}^{N}\left[\alpha_{j} p_{j}+\left(1-\alpha_{j}\right)\right] \\
m(\{2\}) & =\alpha_{2} p_{2} \alpha_{1}\left(1-p_{1}\right) \prod_{j=3}^{N}\left[\alpha_{j} p_{j}+\left(1-\alpha_{j}\right)\right] \\
m(\{i\}) \quad=\alpha_{i} p_{i} \alpha_{i-1}\left(1-p_{i-1}\right) \prod_{j=i+1}^{N}\left[\alpha_{j} p_{j}+\left(1-\alpha_{j}\right)\right] \prod_{k=1}^{i-2}\left[\alpha_{k}\left(1-p_{k}\right)+\left(1-\alpha_{k}\right)\right] \quad \forall i \in[3, N] & \\
m(\{N+1\}) & =\alpha_{N}\left(1-p_{N}\right) \prod_{j=1}^{N-1}\left[\alpha_{j}\left(1-p_{j}\right)+\left(1-\alpha_{j}\right)\right] \\
m([i, j]) \quad & =\alpha_{i-1}\left(1-p_{i-1}\right) \alpha_{j} p_{j} \prod_{m=i}^{j-1}\left(1-\alpha_{m}\right) \prod_{k=j+1}^{N}\left[\left(1-\alpha_{j}\right)+\alpha_{k} p_{k}\right] \prod_{l=1}^{i-2}\left[\alpha_{l}\left(1-p_{l}\right)+\left(1-\alpha_{l}\right)\right] \quad \forall i<j,[i, j] \subset \Theta \\
m(\Theta) & =\prod_{i=1}^{N}\left(1-\alpha_{i}\right),
\end{array}
$$

the rest of the mass being assigned to the empty set.

Example: Let us consider a hypothetical system composed of $N=3$ subsystems. The frame of discernment is then $\Theta=\{1,2,3,4\}$. Without discounting, the different BBAs provided by the classifiers are given by:

$$
\begin{array}{lll}
\text { Classifier } D_{1}: & m_{1}(\{1\})=p_{1} \\
& m_{1}(\{2,3,4\})=1-p_{1} \\
& & \\
\text { Classifier } D_{2}: & m_{2}(\{1,2\}) & =p_{2} \\
& m_{2}(\{3,4\}) & =1-p_{2} \\
& & \\
\text { Classifier } D_{3}: & m_{3}(\{1,2,3\}) & =p_{3} \\
& m_{3}(\{4\}) & =1-p_{3}
\end{array}
$$

The combined BBA is then: 


$$
\begin{array}{ll}
m(\{1\}) & =p_{1} p_{2} p_{3} \\
m(\{2\}) & =\left(1-p_{1}\right) p_{2} p_{3} \\
m(\{3\}) & =\left(1-p_{1}\right)\left(1-p_{2}\right) p_{3} \\
m(\{4\}) & =\left(1-p_{1}\right)\left(1-p_{2}\right)\left(1-p_{3}\right) \\
m(\varnothing) & =1-\sum_{i=1}^{4} m(\{i\}) .
\end{array}
$$

We can notice that if, e.g., $p_{1}=p_{3}=1$ and $p_{2}=0$, there is a contradiction between classifier 1 and classifier 3. Then, applying the formulas, we obtain

$$
m(\{1\})=m(\{2\})=m(\{3\})=0, m(\{4\})=0 \quad m(\varnothing)=1,
$$

which means that there is total conflict between the three classifier outputs.

With discounting, the BBAs given by the classifiers are:

$$
\begin{array}{rll}
\text { Classifier } D_{1}: & m_{1}(\{1\}) & =\alpha_{1} p_{1} \\
& m_{1}(\{2,3,4\}) & =\alpha_{1}\left(1-p_{1}\right) \\
& m_{1}(\{1,2,3,4\}) & =1-\alpha_{1} \\
\text { Classifier } D_{2}: & m_{2}(\{1,2\}) & =\alpha_{2} p_{2} \\
& m_{2}(\{3,4\}) & =\alpha_{2}\left(1-p_{2}\right) \\
& m_{2}(\{1,2,3,4\}) & =1-\alpha_{2} \\
\text { Classifier } D_{3}: & m_{3}(\{1,2,3\}) & =p_{3} \\
& m_{3}(\{4\}) & =\alpha_{3}\left(1-p_{3}\right) \\
& m_{3}(\{1,2,3,4\}) & =1-\alpha_{3}
\end{array}
$$

The combined BBA is:

$$
\begin{aligned}
m(\{1\})= & \alpha_{1} p_{1} \alpha_{2} p_{2} \alpha_{3} p_{3}+\alpha_{1} p_{1}\left(1-\alpha_{2}\right) \alpha_{3} p_{3}+\alpha_{1} p_{1} \alpha_{2} p_{2}\left(1-\alpha_{3}\right)+\alpha_{1} p_{1}\left(1-\alpha_{2}\right)\left(1-\alpha_{3}\right) \\
m(\{2\})= & \alpha_{1}\left(1-p_{1}\right) \alpha_{2} p_{2} \alpha_{3} p_{3}+\alpha_{1}\left(1-p_{1}\right) \alpha_{2} p_{2}\left(1-\alpha_{3}\right) \\
m(\{3\})= & \alpha_{1}\left(1-p_{1}\right) \alpha_{2}\left(1-p_{2}\right) \alpha_{3} p_{3}+\left(1-\alpha_{1}\right) \alpha_{2}\left(1-p_{2}\right) \alpha_{3} p_{3} \\
m(\{4\})= & \alpha_{1}\left(1-p_{1}\right) \alpha_{2}\left(1-p_{2}\right) \alpha_{3}\left(1-p_{3}\right)+\left(1-\alpha_{1}\right) \alpha_{2}\left(1-p_{2}\right) \alpha_{3}\left(1-p_{3}\right) \\
& +\alpha_{1}\left(1-p_{1}\right)\left(1-\alpha_{2}\right) \alpha_{3}\left(1-p_{3}\right)+\left(1-\alpha_{1}\right)\left(1-\alpha_{2}\right) \alpha_{3}\left(1-p_{3}\right) \\
m(\{1,2\})= & \left(1-\alpha_{1}\right) \alpha_{2} p_{2} \alpha_{3} p_{3}+\left(1-\alpha_{1}\right) \alpha_{2} p_{2}\left(1-\alpha_{3}\right) \\
m(\{2,3\})= & \alpha_{1}\left(1-p_{1}\right)\left(1-\alpha_{2}\right) \alpha_{3} p_{3} \\
m(\{3,4\})= & \alpha_{1}\left(1-p_{1}\right) \alpha_{2}\left(1-p_{2}\right)\left(1-\alpha_{3}\right)+\left(1-\alpha_{1}\right) \alpha_{2}\left(1-p_{2}\right)\left(1-\alpha_{3}\right) \\
m(\{1,2,3\})= & \left(1-\alpha_{1}\right)\left(1-\alpha_{2}\right) \alpha_{3} p_{3} \\
m(\{2,3,4\})= & \alpha_{1}\left(1-p_{1}\right)\left(1-\alpha_{2}\right)\left(1-\alpha_{3}\right) \\
m(\Theta)= & \left(1-\alpha_{1}\right)\left(1-\alpha_{2}\right)\left(1-\alpha_{3}\right)
\end{aligned}
$$

the rest of the mass being assigned to the empty set.

\subsection{Decision}

To make the final decision, we compute the pignistic probability of each singleton using (6), and the estimated position $\hat{\theta}$ of the fault is that with the highest pignistic probability: 


$$
\hat{\theta}=\underset{\theta \in \Theta}{\arg \max } \operatorname{Bet} P(\theta)
$$

By convention, $\hat{\theta}=N+1$ is interpreted as absence of fault.

\section{Experimental results}

In this section, the diagnosis system introduced in this paper is assessed using simulated data, and compared to a simple reference approach. The experimental settings will be described in Subsection 5.1 and the results will be reported and discussed in Subsections 5.2 and 5.3, respectively.

\subsection{Experimental settings}

To assess the performances of the method, we considered a track circuit of $N=19$ trimming capacitors and built a database containing 4256 simulated noised signals obtained for different values of the resistance of each capacitor. Among these signals, 608 were fault-free and 3648 had one defective capacitor with a resistance between $\mathrm{r}=1 \Omega$ and $\mathrm{r}=\infty$ (removed capacitor). The data set was split randomly into three subsets (training, validation and test) and the performances were estimated on the test set containing 1064 signals. We have chosen to compare our approach to a basic method using one multilayer perceptron that directly estimates the position of the defective capacitor (position $\mathrm{N}+1$ is used for fault-free case). In such way, we are able to quantify the benefits of our method that builds as many classifiers as trimming capacitors and uses an additional fusion stage to detect and localize a fault in the system.

The following methods were compared:

1. Regression using a multilayer perceptron [4] with one sigmoid hidden layer of 7 neurons and a linear output layer with one neuron. The inputs were the $19 \times 3=57$ parameters $\left(a_{i}, b_{i}, c_{i}\right)$, $i=1, \ldots, 19$ of the Icc curve, and the output was the location of the fault. The virtual location $N+1$ was used when the system was fault-free. This is considered as the reference method. This network was trained to minimize the mean square error between the estimated and the true fault positions on the whole training set. The number of hidden neurons was varied between 3 and 15; the best results on the validation set were obtained with 7 hidden neurons.

2. Fusion with neural networks and decision trees as local classifiers, with the two coding schemes described in Section 4.3, with and without discounting. Overall, this makes 8 different fusionbased methods. The neural network classifiers had one tan-sigmoid hidden layer and a sigmoid output layer with one neuron (Figure 7). Table 1 gives the number of hidden nodes used for each classifier, which was determined by minimizing the error criterion on the validation set. 
Each neural network was trained 100 times with random initializations. The decision tree generation algorithm was the CART method [7].

When discounting was used, the discount rate $1-\alpha_{i}$ for classifier $D_{i}$ was defined from its mean square error $\left(M S E_{i}\right)$ computed on the validation set as:

$$
1-\alpha_{i}=\frac{0.5 M S E_{i}}{\max _{j}\left(M S E_{j}\right)}, \quad i=1 \cdots N
$$

The worst classifier was thus discounted with rate 0.5 , while a perfect classifier such that $M S E_{i}=0$ would not be discounted at all.

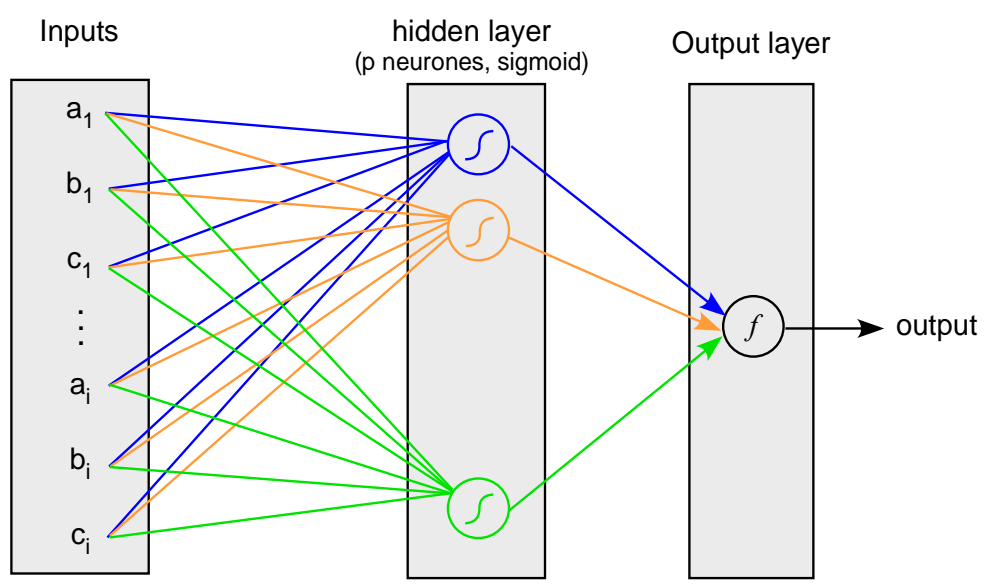

Fig. 7. Architecture of the $i^{\text {th }}$ neural network classifier.

Table 1

Number of hidden nodes for each neural network classifier.

\begin{tabular}{llllll}
\hline$i$ & 1 & 2 & 3 & 4 & $5 \ldots .19$ \\
\hline Number of hidden nodes & 2 & 3 & 4 & 6 & 7 \\
\hline
\end{tabular}

\subsection{Results}

The results of each method were analyzed as shown in Table 2 . We computed the following rates: correct detection (CD), false alarms (FA), non detections (ND) and correct rejections (CR) on the whole data set including defective and fault-free signals. When a fault was detected, we distinguished between correct localizations $(\mathrm{CL}=\mathrm{CD}+\mathrm{CR})$ and false localizations $(\mathrm{FL})$, as it is possible for a fault to be correctly detected but incorrectly localized within the system.

If $N_{0}$ denotes the number of fault-free signals, $N_{I}$ the number of defective signals, and $N_{X}$ the number or cases in category $X$ (where $X$ is $\mathrm{CD}, \mathrm{CR}, \mathrm{FL}$ or FA), different performance measures can be computed as follows: 


$$
\begin{aligned}
t_{C D}=\frac{N_{C D}+N_{C R}+N_{F L}}{N_{0}+N_{1}}, & t_{C L}=\frac{N_{C D}+N_{C R}}{N_{C D}+N_{C R}+N_{F L}} \\
t_{F A}=\frac{N_{F A}}{N_{0}}, & t_{N D}=\frac{N_{N D}}{N_{1}} .
\end{aligned}
$$

The results are shown in Table 3. Results obtained using decision tree classifiers (DT) are also reported to compare them to those of neural networks. The discount rates used to combine the classifier outputs were computed according to (18) and are reported in Figure 8. Except for neural network classifiers using distributed coding, we can see that coefficients $\alpha_{\mathrm{i}}$ tend to decrease with $i$, which means that classifiers are more heavily discounted as $i$ increases, the learning task becoming more complex as we get closer to the receiver.

Table 2

Analysis of the results

\begin{tabular}{cccc}
\cline { 3 - 3 } & & \multicolumn{2}{c}{ Truth } \\
\cline { 3 - 3 } & & Fault position $k$ & No fault \\
\hline \multirow{4}{*}{ Decision } & Fault position $k$ & $C D$ & \multirow{2}{*}{$F A$} \\
\cline { 2 - 3 } & Fault position & $F L$ & $N_{F A}$ \\
& $j \neq k$ & $N_{F L}$ & \\
\cline { 2 - 3 } & No fault & $N D$ & $C R$ \\
& & $N_{N D}$ & $N_{C R}$ \\
\hline
\end{tabular}

Table 3

Performances of the reference regression method as well as the 8 fusion-based methods using neural networks (NN) and decision

\begin{tabular}{|c|c|c|c|c|c|c|c|c|c|}
\hline \multirow{3}{*}{$\begin{array}{l}\text { Rates } \\
\text { (\%) }\end{array}$} & \multirow{3}{*}{$\begin{array}{c}\mathrm{NN} \\
\text { Regression }\end{array}$} & \multirow{2}{*}{\multicolumn{2}{|c|}{$\begin{array}{c}\text { NN } \\
\text { Local coding }\end{array}$}} & \multirow{2}{*}{\multicolumn{2}{|c|}{$\begin{array}{c}\mathrm{NN} \\
\text { Distributed coding }\end{array}$}} & \multirow{2}{*}{\multicolumn{2}{|c|}{$\begin{array}{c}\text { DT } \\
\text { Local coding }\end{array}$}} & \multirow{2}{*}{\multicolumn{2}{|c|}{$\begin{array}{c}\text { DT } \\
\text { Distributed coding }\end{array}$}} \\
\hline & & & & & & & & & \\
\hline & & No disc. & Disc. & No disc. & Disc. & No disc. & Disc. & No disc. & Disc. \\
\hline$t_{\mathrm{CD}}$ & 84.71 & 94.27 & 95.18 & 99.53 & 99.62 & 87.97 & 88.82 & 93.23 & 94.92 \\
\hline $\mathrm{t}_{\mathrm{CL}}$ & 56.12 & 99.8 & 98.28 & 92.26 & 91.89 & 98.93 & 98.42 & 88.61 & 88.42 \\
\hline $\mathrm{t}_{\mathrm{FA}}$ & 59.2 & 1.97 & 1.07 & 1.32 & 0.66 & 2.63 & 1.58 & 2.39 & 1.97 \\
\hline$t_{\mathrm{ND}}$ & 1.17 & 6.36 & 6.36 & 0.33 & 0.33 & 6.16 & 6.36 & 2.41 & 2.52 \\
\hline
\end{tabular}
trees (DT), for the two coding schemes, with and without discounting. 

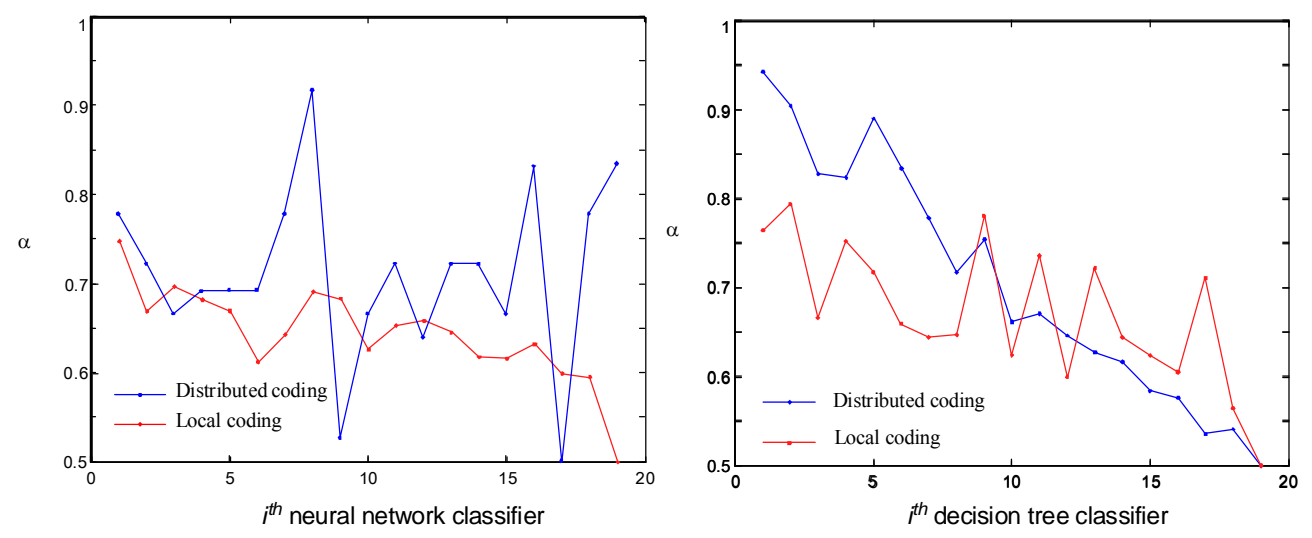

Fig. 8. Coefficients $\alpha_{i}$ used to discount each classifier output (left: neural network, right: decision trees).

\subsection{Discussion}

We can see that, whatever the coding scheme, the fusion methods outperformed the reference regression approach and neural networks yielded better results than decision trees. Not only was the correct detection rate significantly improved, but at the same time the correct localization rate increased to over $90 \%$. In addition, the fusion methods were responsible for only a small number of false alarms ( $\sim 2 \%)$ as compared to the regression method ( $59 \%)$. Indeed, the regression method attempts to simultaneously detect the fault and isolate it within the system. The output is an estimation of the fault position which varied between 1 and $N+1$ where $N$ is the total number of subsystems and $N+1$ is the virtual position used for the fault-free system. In this particular case, if the estimated position is $N+1$ (fault-free system) rather than $N$ (defective system, fault position $=N$ ), this leads to a false alarm. This is the reason why the false alarm rate is high for this method. However, it can be seen that all the methods lead to a small number of non-detections.

Local coding led to a higher non detection rate than distributed coding. This can be explained by the unbalanced number of training instances from each class in the training database. When using local coding, each classifier learnt more 0 s (no fault) than $1 \mathrm{~s}$.

Discounting brought only modest improvement, with a small increase of correct detection rates and a small decrease of false alarm rates, but a slight decrease of correct localizing rates. This is due to the good performances of all the local classifiers whose mean square error was low.

For both learning methods (neural networks and decision trees), local coding induces higher correct localization rates than distributed coding. In order to analyse the magnitude of localization errors, Figure 9 shows the histogram of the errors between the estimated and the true position of the fault in the case of fusion with distributed coding and no discounting. In $91.5 \%$ of the cases, the localization error was equal to 1 , which is satisfactory in this application context. In addition, we estimated the histogram of the serial resistance of the defective capacitor when a fault was not well localized (Figure 10). For $79.2 \%$ of these signals, the capacitor fault was small with a resistance not exceeding $1.5 \Omega$.

Consequently, we can conclude that most faults were well detected by the proposed approach. Localization was also satisfactory even using distributed coding, because almost of the localization errors 
involved small faults that were localized in the closest neighbour of the defective subsystem. Overall, the best results were obtained with the neural network method using distributed coding and discounting.

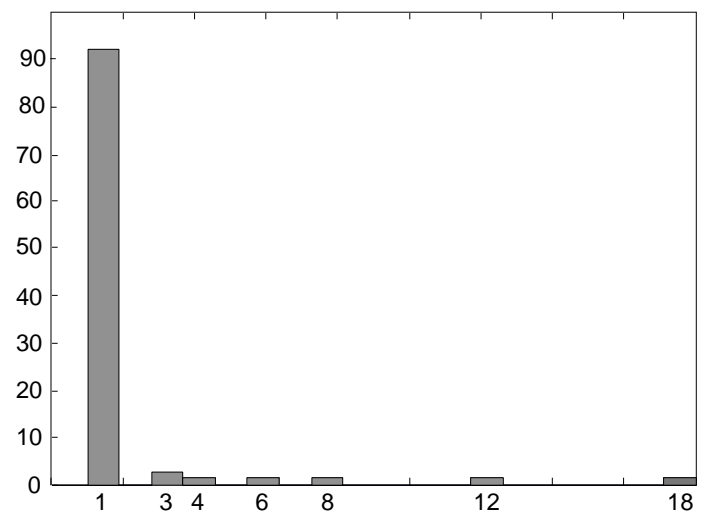

Fig. 9. Histogram of the error between the estimated and the true positions of the fault (NN, Distributed coding)

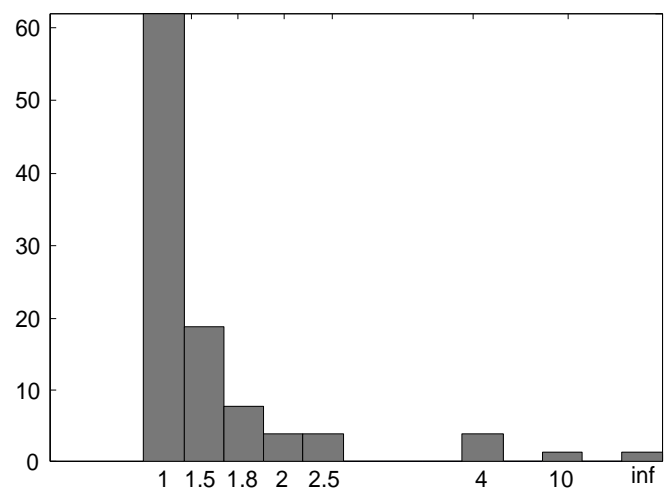

Fig. 10. Histogram of the serial resistance in the case of false localization (NN, Distributed coding)

\section{Limitation of the proposed approach and possible extensions}

The pattern recognition approach presented here gives reliable results but it is only able to detect one defective subsystem (the first one). This might be problematic if it conceals the presence of another defective subsystem that could have a high level of severity. A model-based diagnosis approach based on an electrical transmission line model has been also developed to diagnose track circuits [1] [18] [9]. It seems to be quite relevant, but the correct assessment of defects in the different subsystems is closely linked to accurate tuning of model parameters.

Further studies will be carried out to combine pattern recognition and model-based approaches in order to improve the effectiveness of the final results, especially in the case of multiple defects. The assessment of the severity of the defects is also an interesting subject to consider, particularly in a condition-based maintenance context. 


\section{Conclusion}

This article described an application of pattern recognition and information fusion techniques to fault detection and isolation in a railway track circuits. This device can be considered as a large-scale system that comprises several interconnected and spatially related subsystems. This means that a defective subsystem influences not only its own inspection data but also that of the systems located downstream of it.

The proposed method is based on a classifier fusion approach that builds as many local classifiers as subsystems. The local classifier outputs are then combined using Dempster-Shafer theory, which provides a convenient framework for handling imprecision and uncertainty in decision problems. Two learning strategies for local classifiers have been implemented, which consist in detecting the presence of a fault either on a subsystem or between a subsystem and the transmitter. Both neural networks and decision trees were investigated as local classifiers.

Experiments with simulated data have shown that correct detection rates over $99 \%$ and correct localization over $92 \%$ could be achieved using this approach, which represents a major improvement over the reference method that does not use classifier fusion. Additionally, we have shown that localization errors are small, and incorrectly localized faults generally correspond to a small resistance of the defective capacitor. Overall, the best results were obtained with neural network local classifiers and distributed coding. Although no result with real data could be presented because of lack of labelled signals, the results were judged conclusive enough by the end user to decide the implementation and dissemination of the method.

Further studies are being carried out to handle multiple faults and assess their severity, which may be useful in a predictive maintenance context. The functionality of the system will be extended to discriminate between benign faults, faults that need to be monitored and very serious faults that require immediate maintenance action.

Although the method has been developed in the context of railway track circuit diagnosis, we believe that it can be transferred to other application domains involving the diagnosis of large scale systems composed or linearly organized subsystems, such as other infrastructure networks.

\section{Acknowledgements}

This work was supported by the Infrastructure Department and the Engineering Department of the French National Railway Company (SNCF). The authors thank the anonymous referees for their helpful comments.

\section{References}


[1] M.L. Berova, Development of software tools for simulation and design of jointless track circuits. Ph.D. thesis, The university of Bath.

[2] Y. Bi, D. Bell, H. Wang, G. Guo, K. Greer, Combining Multiple Classifiers Using Dempster's Rule of Combination for Text Categorization, lecture notes in Computer science,31, pp 127-138, 2004.

[3] J. Chen, C. Roberts, P. Weston, Fault detection and diagnosis for railway track circuits using neurofuzzy systems. Control Engineering Practice, 16, 585-596, 2008.

[4] C.M. Bishop, Neural Networks for Pattern Recognition, Oxford university Press edition, 1995.

[5] I. Bloch, Information combination operator for data fusion: a comparative review with classification. IEEE Transactions on Systems, Man, and Cybernetics, 26, p.52-67, 1996.

[6] D. Bonnet, A. Grumbach, V. Labouisse, On the parsimony of the multi-layer perceptrons when processing encoded symbolic variables. Neural Processing Letters, 8(2), p.145-153, 1998.

[7] L. Breiman, J. H. Friedman, R. A. Olshen and C. J. Stone, Classification and Regression Trees, Wadsworth, Belmont, CA, 1984.

[8] A. Debiolles, L. Oukhellou, T. Denoeux, P. Aknin, Output coding of spatially related subclassifiers in evidential framework. Application to the diagnosis of railway track-vehicle system, in Proc. Fusion 2006, Firenze, July 2006.

[9] A. Debiolles, L. Oukhellou, P. Aknin, Combined use of Partial Least Squares regression and neural network for diagnosis tasks, in Proc. Intern. Conf. on Pattern Recognition ICPR'04, Cambridge, 2004.

[10] A. P. Dempster, A generalization of Bayesian inference. Journal of the Royal Statistical Society, 30, p 205-247, 1968.

[11] T. Denœux, M.-H. Masson, B. Dubuisson, Advanced pattern recognition techniques for system monitoring and diagnosis: a survey. Journal Européen des Systèmes Automatisés (RAIRO-APII-JESA), 31(9-10), p. 1509-1539, 1998.

[12] T. Denoeux, Analysis of evidence-theoretic decision rules for pattern classification. Pattern recognition, 30(7):1095-1107, 1997.

[13] D. Dubois, H. Prade, Possibility Theory: An Approach to Computerized Processing of Uncertainty. Plenum Press, New York, November 1988.

[14] R.O. Duda, P.E. Hart, D.G. Stork, Pattern classification, John Wiley and Sons edition, 2001.

[15] Z. Elouedi, K. Mellouli, Ph Smets, Assessing sensor reliability for multi-sensor data fusion within the Transferable Belief Model. IEEE Transactions on Systems, Man, and Cybernetics B, 34(1), p. 782-787, 2004.

[16] L.R. Galina, Combining the results of several neural network classifiers, Neural Networks, vol. 7(5), pp. 777-781, 1994.

[17] J. Gertler: Fault Detection and Diagnosis in Engineering Systems. Marcel Dekker, New York (1998). 
[18] R.J. Hill, D.C. Carpenter, Rail track distributed transmission line impedance and admittance: theoretical modelling and experimental results. IEEE Transactions on Vehicular Technology, 42(4), 225-241, 1993.

[19] T.H. Ho, J.J. Hull, S.N. Srihari, Decision combination in multiple classifier system, IEEE Transactions on Pattern Analysis and Machine Intelligence, 16(1), pp. 66-75, 1994.

[20] Y.S. Huang, C.Y. Suen, A method of combining multiple experts for the recognition of unconstrained handwritten numerals. IEEE Transactions on Pattern Analysis and Machine Intelligence, 17, pp. 90-93, 1995.

[21] R. Isermann, Fault-Diagnosis Systems: An Introduction From Fault Detection To Fault Tolerance, Springer, 2005.

[22] R.W. Jones; A. Lowe; M.J. Harrison, A framework for intelligent medical diagnosis using the theory of evidence, Knowledge-Based Systems, vol. 15(1), p. 77-84(8), 2002, Elsevier.

[23] J. Kittler, Combining classifiers: a theoretical framework. Pattern Analysis and Applications, 1:p.1827, 1998.

[24] J. Korbicz, Advances in fault diagnosis systems, Proc. $10^{\text {th }}$ IEEE Int. Conf. on Methods and Models in Automation and Robotics MMAR, 725-733 (2004).

[25] L. I. Kuncheva, Combining Pattern Classifiers: Methods and Algorithms, John Wiley and Sons edition, 2004.

[26] L. Lam and, C.Y. Suen, Application of Majority Voting to Pattern Recognition: An Analysis of Its Behavior and Performance. IEEE Transactions on Systems, Man, and Cybernetics - Part A: Systems and Humans, 27, pp.553-568, 1997.

[27] C.A. Le., V.N. Huynh, A. Shimazu, Combining classifiers with multi-representation of context in word sense disambiguation, $P A K D D$ 2005, T.B. Ho et al. (Eds.), Springer-Verlag, LNAI 3518, pp. 262268. 2005.

[28] M. R Maurya, R. Rengaswamy and V. Venkatasubramanian, Fault diagnosis using trend analysis: a review and recent developments. Engineering Applications of Artificial Intelligence, 20, p. 133-146, 2007.

[29] D. Mercier, B. Quost and T. Denoeux. Refined modeling of sensor reliability in the belief function framework using contextual discounting, Information Fusion, Vol. 9, p. 246-258, 2008.

[30] R.J. Patton, P. Franck, J. Chen, Fault diagnosis in Dynamic systems: Theory and Applications. Prentice-Hall, New York, 1989.

[31] W. Pedrycz, J.C. Bezdzk, R.J. Hathaway, G.W. Rogers, Two Nonparametric models for fusing heterogeneous fuzzy data, IEEE Transactions on Fuzzy Systems, vol.6 (3), pp. 411-425, 1998.

[32] A. Rakar, D. Juricic and P. Ballé. Transferable belief model in fault diagnosis. Engineering Applications of Artificial Intelligence, 12(5), p. 555-567, 1999. 
[33] R.Rengaswamy, D. Mylaraswamy, Karl-Erik Arzen and V. Venkatasubramanian, A Comparison of Model-Based and Neural Network-Based Diagnostic Methods, Engineering Applications of Artificial Intelligence, 14(6), 805-818, 2002.

[34] D. Ruta, B. Gabrys, An overview of classifier fusion methods, Computing and Information Systems, 7, pp.1-10, 2000.

[35] G. Shafer, A mathematical Theory of Evidence, Princeton University Press, 1976.

[36]P. Smets, Decision making in a context where uncertainty is represented by belief functions. In R.P. Srivastava and T.J. Mock, Editors, Belief Functions in Business Decisions. Physica-Verlag, Heildelberg, 2002.

[37] P. Smets, R. Kennes, The Transferable Belief Model. Artificial Intelligence, vol.66, p.191-234, 1994.

[38] P. Smets, R. Kruse, The Transferable Belief Model for quantified belief representation. In A. Motro and $\mathrm{Ph}$. Smets, editors, Uncertainty in Information Systems: From Needs to Solutions, p. 343-368, Kluwer, Boston, MA, 1997.

[39] P. Smets, The combination of evidence in the Tranferable Belief Model, IEEE transactions on Pattern Analysis and Machine Intelligence, vol.12(5), p. 447-458, 1990.

[40] P. Smets, Belief functions: the disjunctive rule of combination and the Generalized Bayesian theorem. International Journal of Approximate Reasoning, 9, p 1-35, 1993.

[41] F. Valente, H. Hermansky, Combination of Acoustic Classifiers based on Dempster-Shafer Theory of evidence, in Proc. Int. Conf. on Acoustics, Speech, and Signal Processing ICASSP, Honolulu, Hawaï, 2007.

[42] L. Xu, A. Krzyzak, and C.Y. Suen, Methods of combining multiple classifiers and their application to Handwriting Recognition. IEEE Transactions on Systems, Man Cybernetics, 22(3):418-435, May 1992.

[43] G. Yang, X. Wu, Synthesized Fault Diagnosis Method Based on Fuzzy Logic and D-S Evidence Theory, Vol. 4682, pp. 1024-1031, Advanced Intelligent Computing Theories and Applications, 2007, Springer.

[44] L. Zadeh, Fuzzy sets as a basis for a theory of possibility. Fuzzy Sets and Systems, 1(3):3-28, 1978. 